\title{
Métodos de reducción del tiempo de proceso y mejora estructural de capas de SiGe obtenidas por cristalización en fase sólida
}

\author{
A. RODRÍGUEZ, T. RODRÍGUEZ, J. SANGRADOR, C. BALLESTEROS* \\ Departamento de Tecnología Electrónica, E.T.S.I. de Telecomunicación, Universidad Politécnica de Madrid, 28040 Madrid. \\ *Departamento de Física, Escuela Politécnica Superior, Universidad Carlos III, 28911 Leganés (Madrid).
}

\begin{abstract}
Se han investigado dos métodos, implantación iónica y cristalización a dos temperaturas, para reducir el tiempo de proceso y mejorar la estructura de películas policristalinas de SiGe obtenidas por cristalización en fase sólida aplicables en transistores de película delgada. Se partió de capas amorfas de SiGe obtenidas mediante depósito químico en fase vapor a baja presión sobre Si oxidado térmicamente. En ambos casos se estudió la cinética de cristalización y la microestructura de las capas mediante microscopía electrónica de transmisión y difractometría de rayos X. El primer método consistió en la implantación iónica de las muestras con C o F y la posterior cristalización a 600 ${ }^{\circ} \mathrm{C}$. Las muestras implantadas tienen tamaño de grano mayor y más uniforme que las no implantadas y los granos presentan una orientación (111) más acentuada. El segundo método consistió en la cristalización mediante dos recocidos a temperaturas distintas. Las muestras fueron procesadas a $625^{\circ} \mathrm{C}$ mediante procesado térmico rápido durante varios minutos para formar una cierta densidad de núcleos. Seguidamente se cristalizaron las capas a $525^{\circ} \mathrm{C}$ partiendo de los núcleos creados. La duración del proceso se reduce hasta en un $50 \%$. El tamaño de grano es igual o mayor que en las muestras no procesadas previamente y la orientación de los granos no se ve alterada.
\end{abstract}

Palabras Clave: SiGe policristalino, procesado térmico rápido, implantación iónica, cristalización en fase sólida, tamaño de grano, textura.

\section{Methods for process time reduction and structural improvement of SiGe films obtained by Solid Phase Crystallization.}

Two methods, ion implantation and crystallization at two different temperatures, have been investigated to reduce the process time and to improve the structural properties of polycrystalline SiGe films obtained by solid phase crystallization for thin film transistors applications. The starting material was amorphous SiGe films deposited by low pressure chemical vapour deposition on oxidized silicon substrates. The crystallization kinetics and microstructure were analyzed in both cases using transmission electron microscopy and X-ray diffractometry. The first method consisted of the ion implantation with $\mathrm{C}$ or $\mathrm{F}$ and the subsequent crystallization at $600{ }^{\circ} \mathrm{C}$. The implanted samples show greater grain sizes, smaller grain size dispersion and stronger (111) preferred orientation than the unimplanted ones. The second method consisted of the crystallization by sequential annealing at two different temperatures. The samples were processed by rapid thermal annealing at 625 ${ }^{\circ} \mathrm{C}$ for several minutes to form a certain density of crystalline nuclei. The subsequent crystallization at $525{ }^{\circ} \mathrm{C}$ takes place mainly from the previously induced nuclei. The process time is reduced by $50 \%$. The grain size in the samples processed in this way is at least equal than in the samples crystallized without the RTA treatment. This crystallization method does not affect the preferred orientation of the grains.

Keywords: Polycrystalline SiGe, rapid thermal processing, ion implantation, solid phase crystallization, grain size, texture.

\section{INTRODUCCIÓN}

Las capas policristalinas de SiGe obtenidas por cristalización en fase sólida a partir de material amorfo tienen interés para la fabricación de transistores de película delgada (TFT), con aplicaciones en pantallas de cristal líquido [1]. El uso de SiGe presenta como ventaja frente al Si que el tiempo de proceso necesario para la cristalización total de las capas a temperaturas del orden de $550{ }^{\circ} \mathrm{C}$, compatibles con el uso de sustratos de vidrio de bajo coste, es considerablemente menor. No obstante, dicho tiempo sigue siendo del orden de decenas de horas, por lo que se trata de un parámetro a optimizar. En cuanto a la estructura de las capas, hay varios aspectos a considerar. Para reducir los efectos negativos de la presencia de fronteras de grano en las características de los dispositivos, resulta conveniente aumentar el tamaño de grano, tanto en las dos direcciones del plano en el que se encuentra la capa como en la dirección perpendicular a la misma. Es conveniente también que la dispersión del tamaño de grano sea pequeña, con objeto de que las características de los dispositivos sean homogéneas en una superficie grande [2, 3]. La textura de las capas también afecta a las características de los dispositivos, puesto que es conocido que los transistores fabricados usando capas policristalinas con una fuerte orientación preferente de los granos presentan menores voltajes umbral y mayores movilidades de los portadores en el canal que los fabricados usando capas con granos orientados aleatoriamente [2].
En este trabajo se han investigando dos métodos para tratar de reducir el tiempo de proceso necesario para la cristalización en fase sólida de las capas de SiGe y modificar sus propiedades estructurales. Estos métodos se basan en la alteración controlada de los parámetros que afectan al proceso de cristalización en fase sólida, proceso que puede describirse de forma muy simplificada mediante la formación de núcleos cristalinos en las regiones amorfas del material (por nucleación continua aleatoria o por la existencia de centros de nucleación preferente) y su posterior crecimiento hasta la transformación total de la capa amorfa en cristalina.

El primer método, orientado principalmente a mejorar las características morfológicas de las capas, consistió en la implantación de iones de $\mathrm{C}$ o $\mathrm{F}$ en las muestras previamente a su cristalización para aniquilar los posibles centros de nucleación preferente que puedan existir en el volumen de las capas. De este modo se reduce la densidad de núcleos, permitiendo que los núcleos formados crezcan hasta alcanzar un tamaño mayor. Además, la eliminación de núcleos del volumen de la capa posibilita que predomine la nucleación heterogénea en la intercara entre la capa y el sustrato, favoreciendo la aparición de orientaciones preferentes de los granos. En trabajos previos se ha logrado aumentar el tamaño de grano en capas de Si implantando iones de Si o de F $[4,5]$. 
El segundo método está orientado fundamentalmente a disminuir el tiempo de cristalización en fase sólida de las capas. Las velocidades de nucleación y crecimiento tienen la dependencia con la temperatura dada por la expresión de Arrhenius, pero con energías de activación muy distintas. En el caso de Si puro las energías de activación son $5,5 \mathrm{eV}$ y 3,3 eV respectivamente [6]. Esta diferencia en las energías de activación sugiere la cristalización de las capas en dos pasos a temperaturas distintas. En primer lugar se realiza un proceso térmico corto a alta temperatura, con una velocidad de nucleación elevada, para producir de forma controlada una determinada densidad de núcleos. La velocidad de crecimiento de los granos también aumenta, pero en una proporción mucho menor. De este modo es posible realizar el proceso de nucleación sin llegar a producir la cristalización de la capa. A continuación se realiza otro tratamiento térmico a temperatura mucho más baja, de modo que la nucleación adicional sea pequeña, con objeto de cristalizar completamente la capa a partir de los núcleos creados con anterioridad. Esta secuencia de tratamientos debe permitir reducir la duración total del proceso sin que el tamaño de grano (relacionado con la densidad de núcleos existente) disminuya. Resultados previos en capas de $\mathrm{Si}$ indican que la cristalización mediante dos procesos térmicos a temperaturas distintas permite reducir el tiempo de proceso y produce un aumento del tamaño de grano [7].

\section{MÉTODO EXPERIMENTAL}

El material de partida consistió en capas amorfas de $\mathrm{Si}_{1-x} \mathrm{Ge}_{x}$ con fracción de Ge $x=0,33$ y de $100 \mathrm{~nm}$ de espesor obtenidas por depósito químico en fase vapor a baja presión (LPCVD) sobre $\mathrm{SiO}_{2}$. Estos sustratos fueron obtenidos mediante la oxidación térmica de obleas de Si. Se seleccionó esta composición puesto que en trabajos previos se ha comprobado que el volumen de las capas amorfas presenta una elevada densidad de centros de nucleación preferente que hace que los granos de las capas cristalizadas tengan un tamaño inferior al habitual en capas de Si y una orientación aleatoria [8]. En algunos casos se usaron como elemento de comparación muestras con capas de Si puro policristalino obtenido por el proceso convencional de cristalización en fase sólida, usado normalmente para la fabricación de dispositivos. Los detalles sobre el proceso de depósito pueden encontrarse en la literatura [9].

\subsection{Implantación iónica y cristalización}

Para investigar este proceso, las muestras fueron implantadas con iones de carbono ( $30 \mathrm{keV}, 2,0 \times 10^{15} \mathrm{~cm}^{-2}$ ) o flúor $\left(50 \mathrm{keV}, 2,5 \times 10^{15} \mathrm{~cm}^{-2}\right.$ ), de forma que la máxima concentración de impurezas $\left(2 \times 10^{20} \mathrm{~cm}^{-3}\right)$ se situó en la intercara $\mathrm{SiGe/óxido.} \mathrm{Posteriormente} \mathrm{fueron} \mathrm{recocidas}$ en un horno convencional a $600{ }^{\circ} \mathrm{C}$ junto con muestras de SiGe no implantadas que se usaron como referencia. Esta temperatura asegura la cristalización total de todas las muestras, implantadas o no, en un tiempo de decenas de horas, es decir, no excesivamente largo como para que el proceso sea impracticable.

\subsection{Cristalización a dos temperaturas}

En este experimento, las muestras fueron procesadas en primer lugar a $625^{\circ} \mathrm{C}$ mediante procesado térmico rápido (RTA) durante varios minutos para producir la formación de una determinada densidad de núcleos estables, tanto mediante el proceso de nucleación continua como por la nucleación en los posibles centros preferentes existentes. Aunque esta temperatura es superior a la de transición vítrea de los sustratos de vidrio comerciales, se ha comprobado que éstos no resultan dañados en procesos de tan corta duración. La posterior cristalización se realizó a $525{ }^{\circ} \mathrm{C}$ en un horno convencional, siendo la duración del recocido de hasta 100 horas. Esta temperatura puede considerarse baja dentro del margen de temperaturas habitualmente usadas en los procesos de cristalización en fase sólida. La cristalización a esta temperatura debe tener lugar casi exclusivamente a partir de los núcleos creados en el primer proceso mediante RTA, ya que la velocidad de nucleación a $525^{\circ} \mathrm{C}$ es muy baja.

\subsection{Técnicas de caracterización}

Las muestras se caracterizaron mediante difractometría de rayos X en la configuración $\theta-2 \theta$ para determinar la evolución de la fracción cristalizada con el tiempo de proceso, así como la existencia de orientaciones preferentes de los granos y el tamaño de grano en la dirección perpendicular a las capas totalmente cristalizadas. La fracción cristalina $X(t)$ fue obtenida a partir de la intensidad del pico 111 del difractograma, normalizada respecto al valor que toma cuando la película está completamente cristalizada. Cuando fue posible, los resultados experimentales fueron ajustados usando el modelo de Avrami,

$$
X(t)=1-\exp \left\{-\left(\frac{t-t_{o}}{t_{c}}\right)^{a}\right\}
$$

obteniendo a partir de dicho ajuste el tiempo característico $\left(t_{c}\right)$ el tiempo de incubación $\left(t_{0}\right)$ y el exponente de Avrami (a). Estos parámetros fueron utilizados para estimar, a partir de las distribuciones de los tamaños de grano, las velocidades de nucleación $(n)$ y crecimiento (v). Una descripción detallada del propio modelo y del método de realización de los cálculos puede encontrarse en la literatura [6, 10]. La existencia de orientaciones preferentes de los granos se analizó a partir de las intensidades de los picos 111, 220 y 311, presentes en los difractogramas. Para ello se calcularon los cocientes entre las intensidades de los picos $\mathrm{I}_{220} / \mathrm{I}_{111}$ e $\mathrm{I}_{311} / \mathrm{I}_{111}$ y se normalizaron respecto a los valores que deben tener en una muestra en forma de polvo en la que los granos se encuentren orientados al azar. El tamaño de grano en la dirección normal se determinó a partir de la expresión de Scherrer, una vez eliminado el ensanchamiento de los picos debido a efectos instrumentales, obtenido a partir del difractograma de una muestra de silicio monocristalino. Mediante la técnica de microscopía electrónica de transmisión (TEM) se analizó la morfología de los granos en las muestras completamente cristalizadas y se determinó su tamaño en el plano de la capa. Las distribuciones de tamaño de grano obtenidas a partir de las imágenes se ajustaron a una función log-normal, determinando así el tamaño medio de grano y su dispersión. Descripciones más detalladas de estas técnicas experimentales pueden encontrarse en trabajos previos y en las referencias en ellos contenidas [8-10].

\section{RESULTADOS}

\subsection{Implantación iónica y cristalización}

La figura 1 ilustra la cinética de crecimiento de las muestras implantadas y de las usadas como referencia. Se representa en ella la fracción cristalizada en función del tiempo de recocido a $600{ }^{\circ} \mathrm{C}$. También se muestran las curvas de Avrami obtenidas mediante el mejor ajuste de dicho modelo a los datos experimentales. Los parámetros obtenidos a partir del ajuste se resumen en la Tabla I. Las velocidades de nucleación son menores en las muestras de SiGe implantadas con C y F que en la muestra no implantada, debido a la aniquilación de los centros de nucleación preferente que existían en la muestra original. Se observa que la presencia de las impurezas implantadas también afecta, aunque en menor medida, a la velocidad de crecimiento de los granos. La combinación de ambos efectos da como resultado que en el tiempo que dura el proceso de cristalización total se genere una me- 


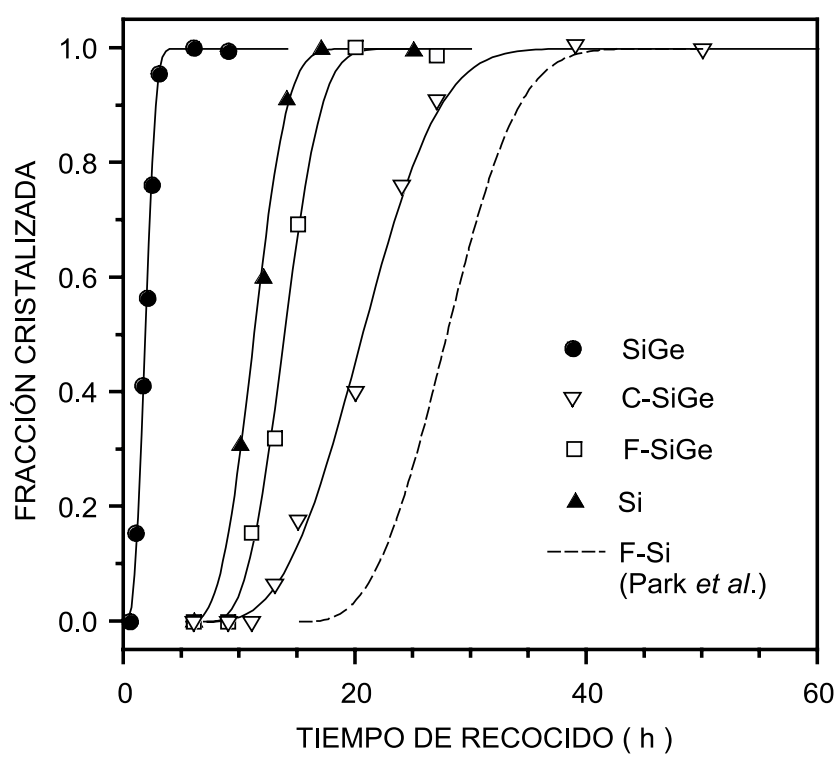

Figura 1. Fracción cristalizada en función del tiempo de recocido a $600{ }^{\circ} \mathrm{C}$ de diversas muestras usadas en los experimentos de implantación iónica. También se representan las curvas de Avrami obtenidas como mejor ajuste a los datos experimentales. La figura incluye la curva correspondiente a la cristalización de Si puro implantado con $\mathrm{F}$ en condiciones similares a las de este trabajo (datos tomados de [5]).

nor densidad de granos, permitiendo que los existentes crezcan hasta alcanzar un tamaño mayor. Otra consecuencia de la disminución de las velocidades de nucleación y crecimiento es que el tiempo necesario para la cristalización de las capas aumenta. Este tiempo es, en el caso peor, menos del doble del tiempo necesario para cristalizar una capa de Si puro a la misma temperatura.

TABLA I. PARÁMETROS DE INTERÉS SOBRE LA CINÉTICA DE CRISTALIZACIÓN Y LA MiCROESTRUCTURA DE LA MUESTRA DE SIGE NO IMPLANTADA, DE LAS IMPLANTADAS CON C Y F Y DE UNA MUESTRA DE SI DE REFERENCIA (VER TEXTO).

\begin{tabular}{|c|c|c|c|c|c|c|c|}
\hline Muestra & $\begin{array}{l}n\left(\times 10^{8}\right) \\
\left(\mathrm{cm}^{-3} \cdot \mathrm{s}^{-1}\right)\end{array}$ & $\begin{array}{c}v\left(\times 10^{-9}\right) \\
\left(\mathrm{cm} \cdot \mathrm{s}^{-1}\right)\end{array}$ & $\frac{I_{220}}{I_{111}}$ & $\frac{I_{311}}{I_{111}}$ & $\underset{(\mathrm{nm})}{\mathrm{d}}$ & $\begin{array}{c}\mathrm{D} \\
(\mu \mathrm{m})\end{array}$ & $\begin{array}{c}\sigma \\
(\mu \mathrm{m})\end{array}$ \\
\hline $\mathrm{Si}$ & 9,3 & 2,8 & 0,18 & 0,10 & 60 & 1,1 & 0,40 \\
\hline SiGe & 190 & 3,4 & 0,51 & 0,33 & 30 & 0,5 & 0,10 \\
\hline SiGe-C & 5,8 & 0,8 & 0,08 & 0,06 & 66 & 1,0 & 0,15 \\
\hline SiGe-F & 8,5 & 2,7 & 0,06 & 0,06 & 83 & 1,1 & 0,30 \\
\hline
\end{tabular}

En la figura 2 se presentan las imágenes obtenidas mediante TEM. En las capas de SiGe implantadas con F el crecimiento de los granos es dendrítico, como ocurre en el caso del Si puro. Las muestras de SiGe no implantadas y las implantadas con $C$ presentan una mayoría de granos con forma más o menos circular o elíptica mezclados con algunos granos dendríticos con pequeño número de ramificaciones. Los granos circulares o elípticos dan como resultado una densidad de defectos en las fronteras y en el interior de los granos menor que la habitual en capas con crecimiento dendrítico.

La figura 3 muestra las distribuciones de los tamaños de grano obtenidas a partir de las imágenes de TEM de las distintas muestras, junto a las curvas resultantes del ajuste de los datos experimentales a la función log-normal. En la Tabla I se incluyen los parámetros de interés obtenidos a partir de dichos ajustes. La implantación de $\mathrm{C}$ o de $\mathrm{F}$ en SiGe previamente a la cristalización da como resultado valores medios del tamaño de grano (D) mayores que el obtenido en la muestra no implantada y similares a los obtenidos en Si puro. La ventaja frente al Si puro es que la dispersión del tamaño de grano $(\sigma)$ en la muestra de SiGe implantada con C $(0,15 \mu \mathrm{m})$ es muy pequeña si se compara con el tamaño medio $(1 \mu \mathrm{m})$.
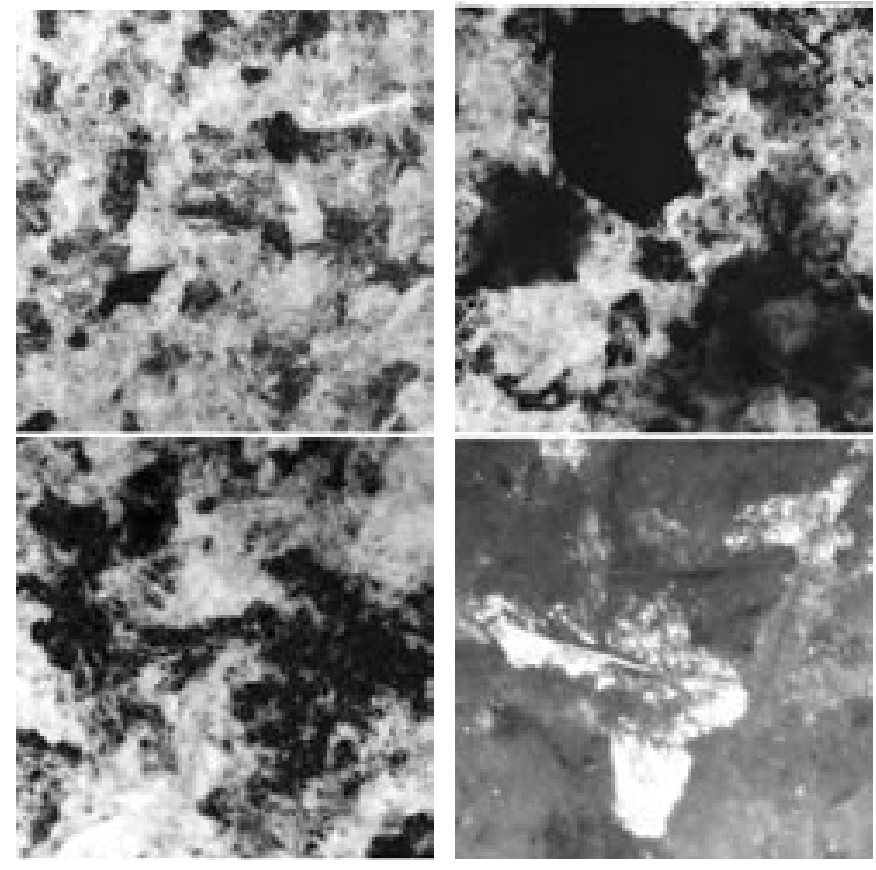

Figura 2. Imágenes TEM de las distintas muestras usadas en los experimentos de implantación iónica y cristalización en fase sólida a 600 ${ }^{\circ} \mathrm{C}$. Las imágenes han sido tomadas en la dirección perpendicular a la superficie de la muestra. La región de la muestra que aparece en cada fotografía tiene $2,0 \mu \mathrm{m}$ de lado.
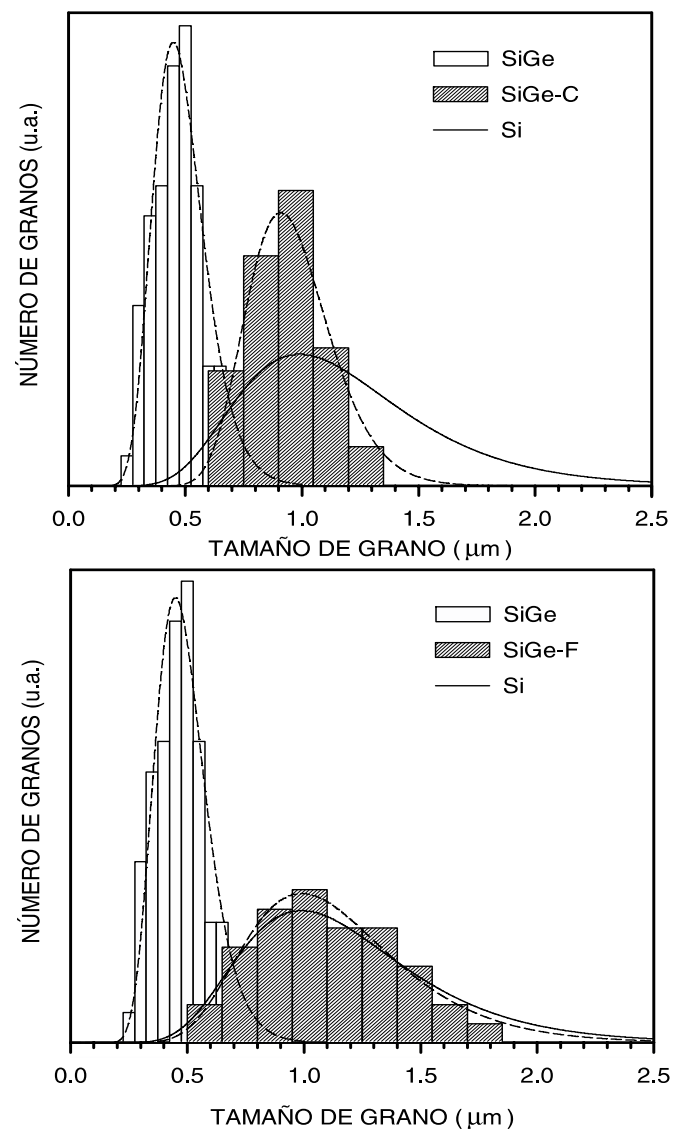

Figura 3. Distribuciones de tamaño de grano y sus ajustes a la función log-normal correspondientes a las muestras implantadas con carbono (a) y con flúor (b) y totalmente cristalizadas a $600^{\circ} \mathrm{C}$. En cada caso se incluyen como referencia las distribuciones obtenidas en las muestras de SiGe no implantado y de Si puro. Los histogramas de la muestra de Si puro han sido omitidos por claridad. 
En la figura 4 están representados los difractogramas de las muestras completamente cristalizadas. Los resultados del análisis de estos difractogramas se resumen en la Tabla I. Las muestras implantadas con carbono o flúor tienen una orientación preferente (111) mucho más marcada que la muestra de SiGe no implantada e incluso que la muestra de Si puro de referencia. El tamaño (d) de los granos con orientación (111), que son los mayoritarios, en la dirección normal a la capa es mayor en las muestras implantadas (tanto con C como con F) que en la muestra no implantada. También es mayor que el obtenido en la muestra de Si puro.

\subsection{Cristalización a dos temperaturas}

La figura 5 resume la cinética de cristalización de las muestras mediante el proceso a dos temperaturas. En ella se ha representado la fracción cristalizada en función de la duración del proceso RTA en dos casos de interés. El primer caso considera el efecto del tratamiento mediante RTA a $625^{\circ} \mathrm{C}$ solamente. Los procesos de hasta 2 minutos de duración no producen cristalización detectable mediante difractometría de rayos X. Tratamientos de mayor duración sí producen una cristalización apreciable de las películas, que llega a ser total si el proceso dura 10 minutos o más. En la figura se ha trazado de forma orientativa una curva de Avrami consistente con los resultados experimentales correspondientes a estos procesos realizados únicamente en RTA. En el segundo caso considerado, las muestras fueron sometidas a un recocido adicional en horno convencional a $525^{\circ} \mathrm{C}$ durante 50 horas. Se aprecia que la muestra procesada previamente mediante RTA durante 2 minutos ha alcanzado la cristalización completa, mientras que la muestra no tratada está cristalizada solamente al 50\%. Se ha comprobado que tras realizar un recocido a $525^{\circ} \mathrm{C}$ durante 100 horas, todas las muestras se encuentran completamente cristalizadas. Este tiempo es el mínimo necesario para cristalizar completamente a $525^{\circ} \mathrm{C}$ la muestra no procesada previamente mediante RTA. Por tanto, la cristalización mediante un doble proceso, primero en RTA durante 2 minutos a 625 ${ }^{\circ} \mathrm{C}$ y después en horno durante 50 horas a $525{ }^{\circ} \mathrm{C}$, supone una reducción de prácticamente 50 horas en el tiempo total de proceso.

El análisis mediante TEM indica que la morfología de los granos es similar en todas las muestras, independientemente del tratamiento realizado. La mayoría de los granos tiene forma circular o elíptica, existiendo algunos con forma dendrítica. El aspecto de las muestras es el mismo que el de la muestra de SiGe cuya imagen TEM aparece en la figura 2. Las distribuciones de tamaño de grano obtenidas a partir de las imágenes tienen el mismo aspecto que la distribución representada en la figura 3-a correspondiente a la muestra de SiGe, con tamaños medios de grano entre 0,4 y 0,6 $\mu \mathrm{m}$. Debido a que las diferencias detectadas entre unas muestras y otras no son significativas, no se han incluido imágenes ni distribuciones.

La figura 6 muestra el tamaño de grano en la dirección perpendicular a la capa, obtenido a partir de las medidas de difracción de rayos X. Se han representado los resultados obtenidos a partir de los picos 111, 220 y 311 de los difractogramas. Estos resultados ponen de manifiesto el efecto del proceso a dos temperaturas en el tamaño de grano de una forma más clara que las imágenes TEM y las distribuciones de tamaños de grano. Para todas las orientaciones se aprecia que el tamaño de grano es mayor en la muestra tratada mediante RTA durante 1 minuto que en la muestra no tratada. En este caso, la densidad de núcleos creados durante los dos procesos a los que se somete a la muestra (el previo mediante RTA y la cristalización a $525^{\circ} \mathrm{C}$ ) es igual o menor que la densidad de núcleos creados durante un único proceso de cristalización de 100 horas de duración a $525^{\circ} \mathrm{C}$, el necesario para cristalizar la muestra no procesada mediante RTA. A partir de las distribuciones

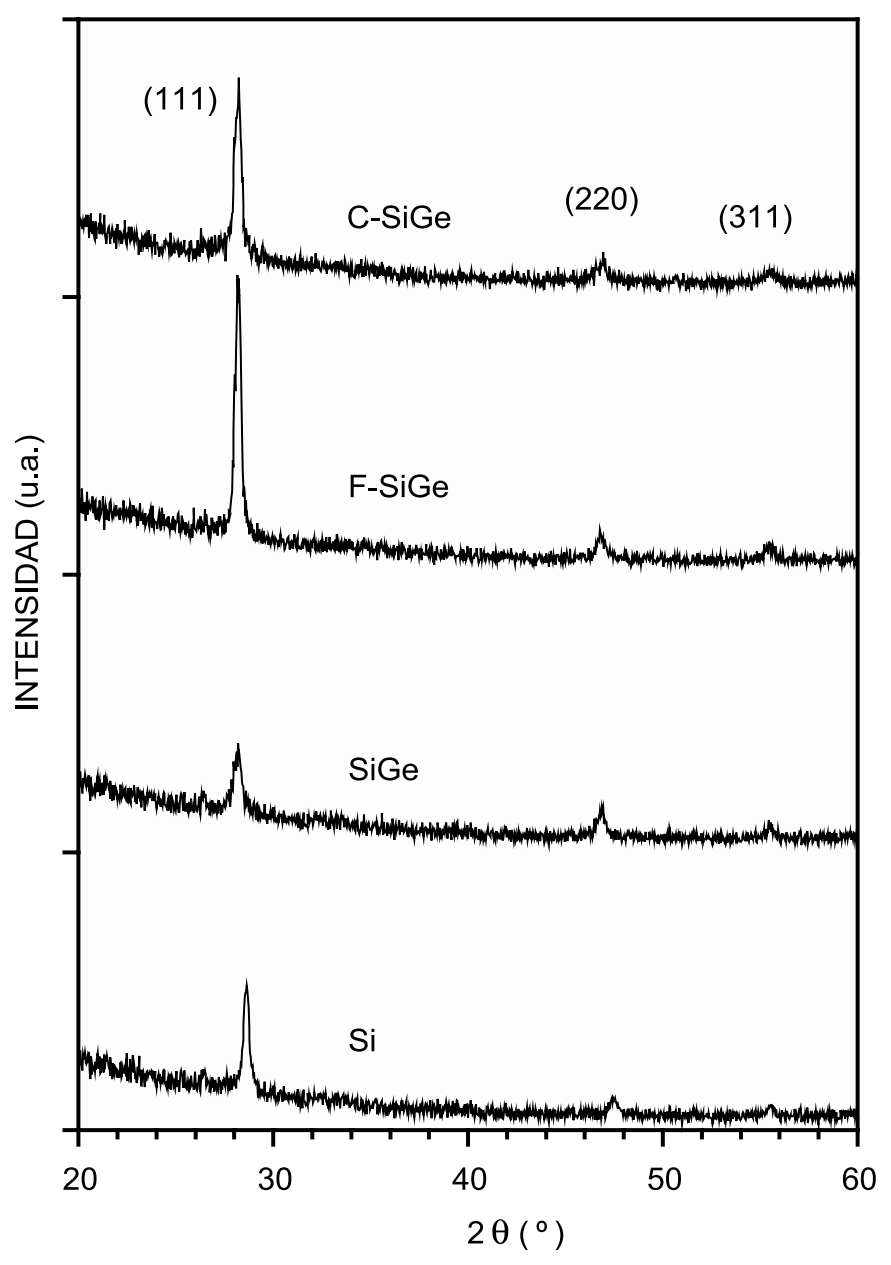

Figura 4. Difractogramas de rayos $\mathrm{X}$ de las distintas muestras usadas en los experimentos de implantación iónica y cristalización en fase sólida a $600{ }^{\circ} \mathrm{C}$.

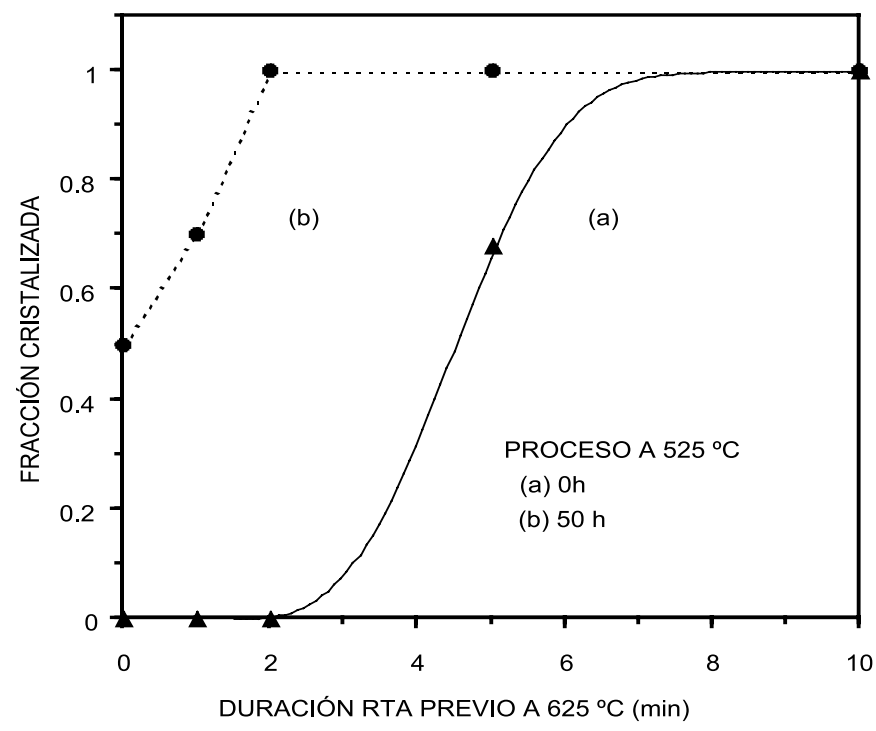

Figura 5. La curva (a) representa la fracción de la muestra de SiGe cristalizada mediante recocidos en RTA a $625^{\circ} \mathrm{C}$ exclusivamente. Se ha representado una curva de Avrami consistente con los resultados experimentales. La curva (b) representa la fracción cristalizada de la muestra de SiGe tras el recocido en horno a $525^{\circ} \mathrm{C}$ durante $50 \mathrm{~h}$ en función de la duración del proceso en RTA a $625^{\circ} \mathrm{C}$ realizado previamente. 
del tamaño de grano y de la curva de Avrami representada en la figura 5 , se estima que la velocidad de nucleación a $625^{\circ} \mathrm{C}$ es del orden de $n=8,75 \times 10^{11} \mathrm{~cm}^{-3} \cdot \mathrm{s}^{-1}$. Del mismo modo, y suponiendo que el tiempo de cristalización a $525^{\circ} \mathrm{C}$ es de unas 100 horas, se obtiene $n=5,10 \times 10^{8} \mathrm{~cm}$ ${ }^{3} \cdot \mathrm{s}^{-1}$ a $525^{\circ} \mathrm{C}$. A partir de estas velocidades de nucleación se deduce que la densidad de granos tras el recocido de 100 horas a $525^{\circ} \mathrm{C}$ es $1,84 \times 10^{14}$ $\mathrm{cm}^{-3}$, mientras que tras la secuencia de recocidos a $625^{\circ} \mathrm{C}$ durante 1 minuto y a $525{ }^{\circ} \mathrm{C}$ durante 50 horas es $1,44 \times 10^{14} \mathrm{~cm}^{-3}$, es decir un $20 \%$ menor aproximadamente. Por ello el tamaño de grano es superior. Si la duración del tratamiento previo es superior a 1 minuto, se observa que el tamaño de grano disminuye respecto al de la muestra no procesada. Este resultado es consecuencia del elevado valor de la velocidad de nucleación a $625^{\circ} \mathrm{C}$, que hace que durante la cristalización total de la muestra mediante el proceso a dos temperaturas se cree una densidad de núcleos mayor que durante el proceso único de 100 horas a $525^{\circ} \mathrm{C}$. Una mayor densidad de núcleos da como resultado necesariamente un tamaño de grano menor. Efectivamente, usando los mismos datos que en el caso anterior se obtiene que la densidad de granos tras los procesos a $625^{\circ} \mathrm{C}$ durante 2 minutos y a $525^{\circ} \mathrm{C}$ durante 50 horas es $1,97 \times 10^{14}$ $\mathrm{cm}^{-3}$. Lo mismo ocurre, obviamente, para duraciones superiores del proceso en RTA. Los resultados sugieren que los tratamientos mediante RTA a $625^{\circ} \mathrm{C}$ de duración inferior a 1 minuto podrían permitir lograr un aumento mayor del tamaño de grano.

Las medidas de difracción de rayos $\mathrm{X}$ indican también que el tratamiento previo mediante RTA seguido de la cristalización a baja temperatura produce granos con la misma orientación que el proceso de cristalización en un solo paso. Dichos granos en ambos casos muestran una ligera tendencia a la orientación preferente (111). Este resultado puede entenderse si se tiene en cuenta que los procesos térmicos realizados no afectan a la localización de los centros de nucleación, que puede tener lugar tanto en el volumen de las capas como en la intercara capa/óxido. Solamente se ven alteradas las velocidades a las que tienen lugar tanto la nucleación como el crecimiento de los granos.

\section{CONCLUSIONES}

(1) La implantación de iones de C en las capas de SiGe amorfas previamente a su cristalización da como resultado una mejora en las propiedades estructurales, que superan a las de las capas de Si usadas normalmente en la fabricación de dispositivos. El tamaño medio de grano aumenta y llega a ser igual que el obtenido en capas de Si puro, pero con una dispersión mucho menor. Los granos conservan la forma circular o elíptica característica de las capas de SiGe, dando como resultado una densidad de defectos en las fronteras y en el interior de los granos menor que la habitual en capas de $\mathrm{Si}$, que muestran crecimiento dendrítico. También aparece una fuerte orientación preferente (111) de los granos, superior a la observada en Si puro. La implantación de iones de $\mathrm{F}$ produce efectos similares excepto en lo referente a la dispersión de los granos, que es similar a la observada en $\mathrm{Si}$, y a la morfología de los mismos, que pasa de ser circular o elíptica a dendrítica.

(2) El procesado térmico rápido de capas amorfas de SiGe previamente a su cristalización a baja temperatura permite reducir en varias decenas de horas el tiempo de recocido necesario para su cristalización total. Las características estructurales de las películas, como la morfología, el tamaño y la orientación de los granos, no se ven significativamente alteradas. Los resultados indican que una adecuada selección de la duración del tratamiento mediante RTA a cada temperatura puede permitir lograr un aumento del tamaño de grano además de reducir el tiempo de proceso.

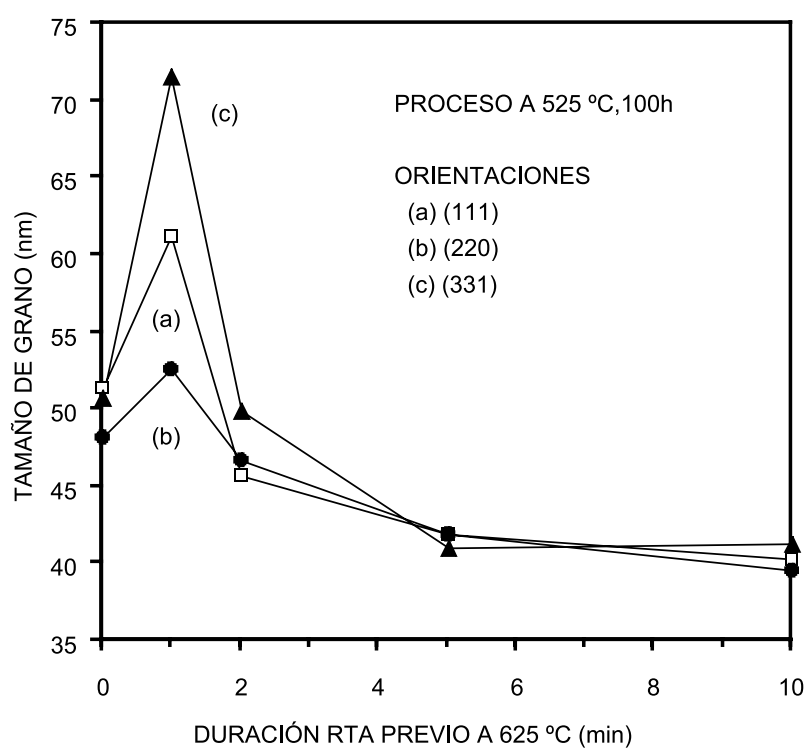

Figura 6. Tamaño de grano en la dirección perpendicular a la superficie de las muestras procesadas a $525^{\circ} \mathrm{C}$ durante $100 \mathrm{~h}$ (totalmente cristalizadas) en función de la duración del proceso RTA previo a 625 ${ }^{\circ} \mathrm{C}$. Los datos han sido obtenidos a partir de los tres picos presentes en los difractogramas de rayos $\mathrm{X}$.

\section{AGRADECIMIENTOS}

Trabajo financiado por la CICYT, Proyecto MAT 99-1214.

\section{REFERENCIAS}

1. J.-W. Kim, M.-K. Ryu, K.-B. Kim, C.-W. Hwang, B. S. Bae, M.-K. Han, S.-J. Kim. "Device characteristics of polycrystalline SiGe thin film transistors grown from disilane and germane source gases". Jpn. J. Appl. Phys. 35 [Part2, 6B] L757-L759 (1996).

2. S. Hasegawa, E. Fujimoto, T. Inokuma, Y. Kurata. “Structure and grain boundary defects of glow-discharge polycrystalline silicon films deposited using disilane". J. Appl. Phys. 77 [1] 357-366 (1995).

3. D. N. Kouvatsos, A. T. Voutsas, M. K. Hatalis. "Polycrystalline silicon thin film transistors fabricated in various solid phase crystallized films deposited on glass substrates". J. Electron. Materials 28 [1] 19 (1999).

4. I.-W. Wu, A. Chiang, M. Fuse, L. Öveçoglu, T. Y. Huang. "Retardation of nucleation rate for grain size enhancement by deep silicon implantation of low-pressure chemical vapor deposited amorphous silicon films". J. Appl. Phys. 65 [10] 4036-4039 (1989).

5. J.-W. Park, D.-G. Moon, B.-T. Ahn, Ho.-B. Im, K. Lee. “Recrystallization of LPCVD amorphous Si films using F implantation". Thin Solid Films 245 [-] 228 (1994).

6. N. Yamauchi, R. Reif. "Polycrystalline silicon thin films processed with silicon ion implantation and subsequent solod-phase crystallization: Theory, experiments, and thin-film transistor applications". J. Appl. Phys. 75 [7] 3235-3257 (1994).

7. V. Subramanian, P. Dankoski, L. Degertekin, B. T. Khuri-Yakub, K. C. Saraswat. "Controlled two-step crystallization for high performance polysilicon TFT's." IEEE Electron Device Lett. 18 [8] 378-381 (1997).

8. J. Olivares, A. Rodríguez, J. Sangrador, T. Rodríguez, C. Ballesteros, A. Kling. "Solid phase crystallization of amorphous SiGe films deposited by LPCVD on $\mathrm{SiO}_{2}$ and glass". Thin Solid Films 337 [1-2] 51-54 (1999).

9. J. Olivares, J. Sangrador, A. Rodríguez, T. Rodríguez. “Effect of deposition parameters on the characteristics of SiGe films grown by LPCVD using $\mathrm{Si}_{2} \mathrm{H}_{6}$ and $\mathrm{GeH}_{4}$ source gases." J. Electrochem. Soc. 148 [10] C685-C689 (2001).

10. J. Olivares, A. Rodríguez, J. Sangrador, T. Rodríguez, P. Martín, J. Jiménez, C. Ballesteros, M. Castro. "Influence of the alloy composition on the thermodynamic parameters of nucleation and growth of SiGe." Mat. Res. Soc. Symp. Proc. 580 [-] 47-52 (2000).

Recibido: 1.2 .03

Aceptado: 30.11 .03 